\title{
Breaking and Fixing of an Identity Based Multi-Signcryption Scheme
}

\author{
S. Sharmila Deva Selvi, S. Sree Vivek * and C. Pandu Rangan ${ }^{\star}$ \\ Theoretical Computer Science Lab, \\ Department of Computer Science and Engineering, \\ Indian Institute of Technology Madras, India. \\ \{sharmila, svivek, prangan\}@cse.iitm.ac.in
}

\begin{abstract}
Signcryption is a cryptographic primitive that provides authentication and confidentiality simultaneously in a single logical step. It is often required that multiple senders have to signcrypt a single message to a certain receiver. Obviously, it is inefficient to signcrypt the messages separately. An efficient alternative is to go for multi-signcryption. The concept of multi-signcryption is similar to that of multi-signatures with the added property - confidentiality. Recently, Jianhong et al. proposed an identity based multi-signcryption scheme. They claimed that their scheme is secure against adaptive chosen ciphertext attack and it is existentially unforgeable. In this paper, we show that their scheme is not secure against chosen plaintext attack and is existentially forgeable, we also provide a fix for the scheme and prove formally that the improved scheme is secure against both adaptive chosen ciphertext attack and existential forgery.
\end{abstract}

Keywords. Identity Based Cryptography, Signcryption, Cryptanalysis, Multi-Signcryption, Bilinear Pairing, Provable Security, Random Oracle Model.

\section{Introduction}

Secure message transmission over an insecure channel like internet requires both confidentiality and authenticity. An encryption scheme is used to achieve confidentiality and digital signature is used to achieve unforgeability. Digital signcryption scheme is a cryptographic primitive that achieves both these properties together in an efficient way. In 1997, Zheng [17] proposed the first digital signcryption scheme with the aforementioned properties, lower computational cost and communication overhead than signing then encrypting $(S t E)$ or encrypting then signing $(E t S)$ the message (i.e, signing and encrypting the same message independently). Since then, many signcryption schemes were proposed. Baek et al. [2] gave the formal security model for digital signcryption schemes and provided the security proof for Zheng's [17] scheme in the random oracle model. In 1984, Shamir [14] introduced the concept of identity based cryptography and proposed the first identity based signature scheme. The idea of identity based cryptography is to enable an user to use any arbitrary string that uniquely identifies him as his public key. Identity based cryptography serves as an efficient alternative to Public Key Infrastructure (PKI) based systems.

By combining identity based cryptography and signcryption, Malone-Lee [10] proposed the first identity based signcryption scheme. But Libert et al. [9] pointed out that Malone-Lee's scheme [10] is not semantically secure, since the signature of the message is visible in the signcryption. Chow et al. [6] proposed another identity based signcryption scheme that provides both public verifiability and forward security. Boyen [5] proposed yet another identity based signcryption scheme with ciphertext anonymity in the random oracle model. Following that, Libert et al. [8] modified Boyen's security model to a PKI based signcryption scheme and proposed a scheme as well. They claimed that their signcryption scheme was semantically secure against adaptive chosen ciphertext attacks, ciphertext anonymity and key invisibility. However, Tan [15] showed that the scheme by Libert et al. [8] did not satisfy the above properties. Till date, the most efficient identity based signcryption scheme was the one proposed by Barreto et al. [3].

\footnotetext{
* Work supported by Project No. CSE/05-06/076/DITX/CPAN on Protocols for Secure Communication and Computation sponsored by Department of Information Technology, Government of India
} 
Multi-signatures allow multiple signers to jointly authenticate a message using a single compact signature. Gentry et al [7] were the first to propose a provably secure identity based multi-signature scheme based on bilinear pairing. Following that, Bellare et al. [4] gave a provably secure identity based multi-signature scheme based on RSA. Multi-receiver signcryption scheme provides an efficient way to signcrypt a single message to an ad-hoc group of receivers chosen by the sender. In contrast to multi-receiver signcryption schemes, multi-signcryption schemes can be employed when multiple senders want to signcrypt a single message to a single receiver. Informally, a multi-signcryption scheme can be considered as a multi-signature scheme with message confidentiality. Jianhong et al. [16] were the first to propose an identity based multi-signcryption scheme. There are a few multi-signcryption schemes available in the literature $[12,11,13]$ but to the best of our knowledge Jianhong et al.'s [16] scheme is the only identity based multi-signcryption scheme available to date.

Multi-signcryption schemes can be directly used in many applications, such as E-Business - for a joint signing of confidential contracts between two or more organizations, or E-Government - to signcrypt an electronic legal document by a number of higher authorities, or in membership - for access right authentication of confidential resources. In all these applications if the signcryptions are performed individually by the group of senders, the cost involved in unsigncryption is high. Multi-signcryption enables to perform unsigncryption with the cost equal to a single unsigncryption irrespective of the number of senders.

Our Contribution. In this paper, we point out that Jianhong et al.'s [16] scheme is insecure with respect to chosen plaintext attack and is existentially forgeable. We also propose an improvement to Jianhong et al.'s [16] identity based multi-signcryption scheme and formally prove the confidentiality of the scheme against adaptive chosen ciphertext and identity attack. We give a new security model for unforgeability, which is adapted from Bellare et al.'s security model for identity based multi-signature schemes. Thus our scheme turns out to be the first provably secure identity based multi-signcryption scheme. We also give the complexity figure of the improved scheme.

\section{Preliminaries}

\subsection{Bilinear Pairing}

Let $\mathbb{G}_{1}$ be an additive cyclic group generated by $P$, with prime order $q$, and $\mathbb{G}_{2}$ be a multiplicative cyclic group of the same order $q$. A bilinear pairing is a map $\hat{e}: \mathbb{G}_{1} \times \mathbb{G}_{1} \rightarrow \mathbb{G}_{2}$ with the following properties.

- Bilinearity. For all $P, Q, R \in \mathbb{G}_{1}$,

- $\hat{e}(P+Q, R)=\hat{e}(P, R) \hat{e}(Q, R)$

- $\hat{e}(P, Q+R)=\hat{e}(P, Q) \hat{e}(P, R)$

- $\hat{e}(a P, b Q)=\hat{e}(P, Q)^{a b}\left[\right.$ Where $\left.a, b \in_{R} \mathbb{Z}_{q}^{*}\right]$

- Non-Degeneracy. There exist $P, Q \in \mathbb{G}_{1}$ such that $\hat{e}(P, Q) \neq I_{\mathbb{G}_{2}}$, where $I_{\mathbb{G}_{2}}$ is the identity element of $\mathbb{G}_{2}$.

- Computability. There exists an efficient algorithm to compute $\hat{e}(P, Q)$ for all $P, Q \in \mathbb{G}_{1}$.

\subsection{Computational Assumptions}

In this section, we review the computational assumptions related to bilinear maps that are relevant to the protocol we discuss.

\section{Computation Diffie-Hellman Problem (CDHP)}

Definition 1. Given $(P, a P, b P) \in \mathbb{G}_{1}^{3}$ for unknown $a, b \in \mathbb{Z}_{q}^{*}$, the $C D H$ problem in $\mathbb{G}_{1}$ is to compute abP. Definition. The advantage of any probabilistic polynomial time algorithm $\mathcal{A}$ in solving the CDH problem in $\mathbb{G}_{1}$ is defined as

$$
A d v_{\mathcal{A}}^{C D H}=\operatorname{Pr}\left[\mathcal{A}(P, a P, b P)=a b P \mid a, b \in \mathbb{Z}_{q}^{*}\right]
$$

The $C D H$ Assumption is that, for any probabilistic polynomial time algorithm $\mathcal{A}$, the advantage $A d v_{\mathcal{A}}^{C D H}$ is negligibly small. 


\subsection{Computational Bilinear Diffie-Hellman Problem (CBDHP)}

Definition 2. Given $(P, a P, b P, c P) \in \mathbb{G}_{1}^{4}$ for unknown $a, b, c \in \mathbb{Z}_{q}^{*}$, the CBDHP in $\mathbb{G}_{1}$ is to compute $\hat{e}(P, P)^{a b c} \in \mathbb{G}_{2}$.

The advantage of any probabilistic polynomial time algorithm $\mathcal{A}$ in solving the CBDHP in $\mathbb{G}_{1}$ is defined as

$$
A d v_{\mathcal{A}}^{C B D H P}=\operatorname{Pr}\left[\mathcal{A}(P, a P, b P, c P)=\hat{e}(P, P)^{a b c} \mid a, b, c \in \mathbb{Z}_{q}^{*}\right]
$$

The $C B D H$ Assumption is that, for any probabilistic polynomial time algorithm $\mathcal{A}$, the advantage $A d v_{\mathcal{A}}^{C B D H P}$ is negligibly small.

\subsection{Identity-Based Multi-Signcryption}

A generic Identity-Based Multi-Signcryption scheme for signcrypting a single message from $n$ senders to a single recipient consists of the following probabilistic polynomial time algorithms:

- $\operatorname{Setup}(\kappa)$. Given a security parameter $\kappa$, the Private Key Generator (PKG) generates the public parameters params and master secret key msk of the system.

- Key Extract $\left(I D_{i}\right)$. Given an identity $I D_{i}$ of an user, the PKG computes the corresponding private key $S_{i}$ and transmits it to owner in a secure way.

- Signcryption $\left(m, \mathcal{L}=\left\{I D_{1}, \ldots, I D_{n}\right\}, I D_{X}, S_{1}, \ldots, S_{n}\right)$. To signcrypt a message $m$ to the receiver $I D_{X}$, the set of senders $\mathcal{L}$ with identity $I D_{1}, \ldots, I D_{n}$ and private key $S_{1}, \ldots, S_{n}$ run this algorithm to obtain the signcryption $\sigma$.

- Unsigncryption $\left(\sigma, \mathcal{L}=\left\{I D_{1}, \ldots, I D_{n}\right\}, I D_{X}, S_{X}\right)$. When the receiver $X$ with identity $I D_{X}$ and private key $S_{X}$ receives the signcrypted ciphertext $\sigma$ from a set of senders $\mathcal{L}$ with identities $\mathcal{L}=$ $\left\{I D_{1}, \ldots, I D_{n}\right\}, X$ executes this algorithm to obtain either the plain text $m$ or a message invalid with respect to the validity of $\sigma$.

\subsection{Security Model for Identity-Based Multi-Signcryption}

The notion of semantic security of public key encryption was extended to identity-based signcryption scheme by Malone-Lee in [10]. This was later modified by Sherman et al. in [6] which incorporates indistinguishability against adaptive chosen ciphertext and identity attacks and existential unforgeability against adaptive chosen message and identity attacks. We describe below the security models for confidentiality of an identity-based multi-signcryption scheme against adaptively chosen ciphertext and identity attack, and provide a new model for unforgeability based on unforgeability model of Bellare et al.'s [4] for identity based multi-signature scheme. These are the strongest security notions for this problem. It is to be noted that Jianhong et al. [16] have proved confidentiality in the chosen identity model where as we prove our scheme in the adaptive chosen identity model which is considered to be stronger than chosen identity model [5].

Confidentiality: An identity based multi-signcryption scheme is indistinguishable against adaptive chosen ciphertext attacks (IND-CCA2), if no polynomially bounded adversary $\mathcal{A}$ has a non-negligible advantage in the following game between the challenger $\mathcal{C}$ and $\mathcal{A}$ :

Setup: The challenger $\mathcal{C}$ runs this algorithm to generate the master public and private keys, params and $m s k$ respectively. $\mathcal{C}$ gives params to $\mathcal{A}$ and keeps the master private key $m s k$ secret from $\mathcal{A}$.

Phase 1: $\mathcal{A}$ performs a series of queries in an adaptive fashion in this phase. The queries allowed are given below:

Key Extract queries: $\mathcal{A}$ chooses an identity $I D_{i}$ and gives it to $\mathcal{C}$. $\mathcal{C}$ computes the corresponding private key $S_{i}$ and sends it to $\mathcal{A}$.

Signcryption queries: $\mathcal{A}$ produces a list of senders $\mathcal{L}=\left\{I D_{1}, \ldots, I D_{n}\right\}$, the recipient identity $I D_{X}$ and a message $m$ to $\mathcal{C}$. $\mathcal{C}$ computes the signcryption $\sigma$ of the message $m$ with $\mathcal{L}$ as the group of senders, $I D_{X}$ as the receiver and sends $\sigma$ to $\mathcal{A}$.

Unsigncryption queries: Given a ciphertext $\sigma=\operatorname{Signcryption}\left(m, \mathcal{L}=\left\{I D_{1}, \ldots, I D_{n}\right\}, I D_{X}, S_{1}, \ldots, S_{n}\right)$, 
$\mathcal{C}$ unsigncrypts $\sigma$ with the receiver private key $S_{X}$, the list of sender identities $\mathcal{L}$ and returns the corresponding message to $\mathcal{A}$ if $\sigma$ is a valid signcryption from the list if senders $\mathcal{L}$ to the receiver $I D_{X}$, else, $\mathcal{C}$ returns "invalid".

Challenge: At the end of Phase $1, \mathcal{A}$ sends to $\mathcal{C}$, plaintexts $m_{0}$ and $m_{1}$ of equal length, a receiver identity $I D_{X}$ and a sender list $\mathcal{L}=\left\{I D_{1}, \ldots, I D_{n}\right\}$. Here, $\mathcal{A}$ should have created the public key corresponding to the user in the sender list $\mathcal{L}$ and the receiver $I D_{X}$. However, $\mathcal{A}$ should not have queried the private key of $I D_{X}$ to the key extract oracle. Now, $\mathcal{C}$ chooses $b \in_{R}\{0,1\}$ and computes the challenge signcryption $\sigma^{*}$ on the message $m_{b}$ with $\mathcal{L}$ as the group of senders, $I D_{X}$ as the receiver and returns $\sigma^{*}$ to $\mathcal{A}$.

Phase 2: $\mathcal{A}$ can perform polynomially bounded number of queries adaptively again as in Phase 1 but $\mathcal{A}$ cannot ask the key extraction query on the receiver identity $I D_{X}$ or cannot ask the unsigncryption query on the challenge signcryption $\left(\sigma^{*}, I D_{X}\right)$.

Guess: $\mathcal{A}$ outputs a bit $b^{\prime}$ and wins the game if $b^{\prime}=b$.

The advantage of $\mathcal{A}$ is defined as $A d v_{\mathcal{A}}=\left|2 \operatorname{Pr}\left[b^{\prime}=b\right]-1\right|$, where $\operatorname{Pr}\left[b^{\prime}=b\right]$ denotes the probability that $b^{\prime}=b$.

Existential Unforgeability: Jianhong et al. [16] did not provide the formal security model for unforgeability of identity based multi-signcryption scheme but they claim that, a slightly altered security model of Malone-Lee et al. [10] has been adapted for their scheme. Bellare et al., in [4] have given two security notions for unforgeability of identity based multi-signature schemes, namely, single-signer security and multisigner security, and have proved that single-signer security implies multi-signer security [4]. We extend the notion of single-signer security of Bellare et al. to prove the unforgeability of our improved identity based multi-signcryption scheme.

An identity based multi-signcryption scheme is claimed to be existentially unforgeable under chosen message attack (EUF-CMA), if any polynomially bounded forger $\mathcal{F}$ has a negligible advantage in the following game:

Setup: The challenger $\mathcal{C}$ runs the $\boldsymbol{S e t u p}$ algorithm to generate the master public and private keys, params and $m s k$ respectively. $\mathcal{C}$ gives params to $\mathcal{F}$ and keeps the master private key msk secret from $\mathcal{F}$.

Training Phase: $\mathcal{F}$ asks polynomial number of queries to the various oracles provided by $\mathcal{C}$, without any restrictions.

Forgery: At the end of the Training Phase, $\mathcal{F}$ chooses a message $m$ and produces a signcryption $\sigma^{*}$ on $m$ with $\mathcal{L}=\left\{I D_{1}, \ldots, I D_{n}\right\}$ as the list of sender identities and $I D_{X}$ as the receiver identity. $\mathcal{F}$ wins the game if the private keys of at least one of the user in the list of senders $\mathcal{L}$ was not queried during Training Phase, $\sigma^{*}$ is a valid signcryption and $\sigma^{*}$ is not the output of any previous queries to the Signcryption Oracle with $m$ as the message, $\mathcal{L}$ as the sender list and $I D_{X}$ as the receiver. (note that the private key $S_{X}$ of the receiver $I D_{X}$ can be queried by $\mathcal{F}$ during the Training Phase.)

\section{Review and Attack of Jianhong et al.'s Identity Based Multi-Signcryption Scheme (JJ-IBMSC)}

In this section we review the identity based multi-signcryption scheme of Jianhong et al.'s (JJ-IBMSC) presented in [16]. We also show that [16] does not provide both confidentiality and unforgeability.

\subsection{Review of the scheme}

This scheme has the following four algorithms.

1. Setup: Given $\kappa$ the security parameter and $l$ the length of the message, the PKG chooses two groups $\mathbb{G}_{1}$ and $\mathbb{G}_{2}$ of prime order $q>2^{\kappa}$, a bilinear map $\hat{e}: \mathbb{G}_{1} \times \mathbb{G}_{1} \rightarrow \mathbb{G}_{2}$ and a random generator $P$ of $\mathbb{G}_{1}$. It then chooses a master private key $s \in_{R} \mathbb{Z}_{q}^{*}$, a system-wide public key $P_{p u b}=s P$ and three cryptographic hash functions $H_{1}:\{0,1\}^{*} \rightarrow \mathbb{G}_{1}, H_{2}: \mathbb{G}_{2} \rightarrow\{0,1\}^{l}$ and $H_{3}:\{0,1\}^{*} \rightarrow \mathbb{Z}_{q}^{*}$. The public parameters Params $=\left\langle\mathbb{G}_{1}, \mathbb{G}_{2}, P, \hat{e}, H_{1}, H_{2}, H_{3}, P_{\text {pub }}, \kappa\right\rangle$.

2. Key Extract: Given an identity $I D_{i} \in\{0,1\}^{*}$, the PKG does the following to extract the private key corresponding to $I D_{i}$ : 
- Computes $Q_{i}=H_{1}\left(I D_{i}\right) \in \mathbb{G}_{1}$ and

- Computes the private key $S_{i}=s Q_{i}$.

3. Signcryption: Given a message $m$, a receiver identity $I D_{X}$ and a list of $n$ sender identities $\mathcal{L}=$ $\left\{I D_{1}, \ldots, I D_{n}\right\}$ Each sender $i$ executes the following steps:

- Chooses $x_{i} \in_{R} \mathbb{Z} q^{*}$ and computes $R_{i}=x_{i} P$.

- Computes $\omega_{i}=\hat{e}\left(P_{p u b}, Q_{X}\right)^{x_{i}}$.

- Sends $\left\langle R_{i}, \omega_{i}\right\rangle$ to the other senders through a secure channel.

- After receiving all other senders $\left\langle R_{i}, \omega_{i}\right\rangle$ values, each sender computes $\omega=\prod_{j=1}^{n} \omega_{j}, c=H_{2}(\omega) \oplus m$, $R=\sum_{j=1}^{n} R_{j}$ and $T_{i}=x_{i} H_{1}(m)+H_{3}(R) S_{i}$.

The resultant signcryption is $\sigma=\langle c, T, R, \mathcal{L}\rangle$, where $T=\sum_{j=1}^{n} T_{j}$.

4. Unsigncryption: Given $\sigma=\langle c, T, R, \mathcal{L}\rangle$, the receiver with identity $I D_{X}$ does the following:

- Computes $\omega^{\prime}=\hat{e}\left(R, S_{X}\right)$.

- Retrieves the message $m=H_{2}\left(\omega^{\prime}\right) \oplus c$.

- Accepts the message if $\hat{e}(T, P) \stackrel{?}{=} \hat{e}\left(R, H_{1}(m)\right) \hat{e}\left(P_{p u b}, \sum_{j=1}^{n} Q_{j}\right)^{H_{3}(R)}$

\subsection{Attacks on Jianhong et al.'s Identity Based Multi-Signcryption Scheme (JJ-IBMSC)}

We launch two different attacks on the scheme to show both the weakness in confidentiality as well as unforgeability.

Attack on Confidentiality: During the confidentiality game the adversary $\mathcal{A}$ interacts with the challenger $\mathcal{C}$ in Phase 1 by performing various queries on Hash, Key Extraction, Signcryption and Unsigncryption oracles. At the end of Phase 1, $\mathcal{A}$ choose two messages $m_{0}$ and $m_{1}$, a list of sender identities $\mathcal{L}=\left\{I D_{1}, \ldots, I D_{n}\right\}$ and a targeted receiver identity $I D_{X}$ on which $\mathcal{A}$ wants to be challenged and sends them to $\mathcal{C}$ (note that, $\mathcal{A}$ should not have queried the private key for the targeted receiver identity $I D_{X}$ throughout the game but is allowed to query the private keys corresponding to all the senders in $\mathcal{L}$ ). Now, $\mathcal{C}$ chooses a bit $b \in_{R}\{0,1\}$ and generates a challenge signcryption $\sigma^{*}=\left\langle c^{*}, T^{*}, R^{*}\right\rangle, \mathcal{L}$ and sends $\sigma^{*}$ to $\mathcal{A}$.

We now show that up on receiving the challenge signcryption $\sigma^{*}, \mathcal{A}$ can perform the following to check whether $\sigma^{*}$ is a valid signcryption of message $m_{0}$ or $m_{1}$.

- Computes $\mathcal{H}_{0}=H_{1}\left(m_{0}\right)$.

- Checks whether $\hat{e}(T, P) \stackrel{?}{=} \hat{e}\left(R, \mathcal{H}_{0}\right) \hat{e}\left(P_{p u b}, \Sigma_{j=1}^{n} Q_{j}\right)^{H_{3}(R)}$.

- If the above check passes then $\sigma^{*}$ is the signcryption of $m_{0}$, otherwise $\sigma^{*}$ is the signcryption of the message $m_{1}$.

This shows that the scheme is not even secure against chosen plaintext attack because on receiving the challenge signcryption $\sigma^{*}, \mathcal{A}$ is capable of distinguishing whether $\sigma^{*}$ is a signcryption of $m_{0}$ or $m_{1}$, without any further interaction with $\mathcal{C}$.

Remark 1: This attack is possible because $\mathcal{A}$ knows the messages $m_{0}$ and $m_{1}$ during the confidentiality game and during unsigncryption, the message is directly used for the verification test $\hat{e}(T, P) \stackrel{?}{=}$ $\hat{e}\left(R, H_{1}(m)\right) \hat{e}\left(P_{p u b}, \sum_{j=1}^{n} Q_{j}\right)^{H_{3}(R)}$. Therefore, $\mathcal{A}$ can check the validity of $\sigma^{*}$ with respect to both the messages and find out for which message the equation holds.

Attack on Unforgeability: The forger $\mathcal{F}$ aims to generate the signcryption of a message $m$ on a list of senders $\mathcal{L}^{*}$ to a receiver $I D_{Y}$. Here the forger does not know the private key of any of the identities in $\mathcal{L}^{*}$. In order to achieve this, $\mathcal{F}$ first selects a set of identities $\mathcal{L}^{\prime}=\left\{I D_{1}, \ldots, I D_{k^{\prime}}\right\}$, where $\mathcal{F}$ knows the private key corresponding to all the identities in $\mathcal{L}^{\prime} . \mathcal{F}$ then sets $\mathcal{L}=\mathcal{L}^{*} \cup \mathcal{L}^{\prime}$. Now, $\mathcal{F}$ sends a message $m$, the list $\mathcal{L}$ and an identity $I D_{X}\left(\right.$ where $\left.I D_{X} \neq I D_{Y}\right)$ to the challenger $\mathcal{C}$ and obtains a signcryption $\sigma=\langle c, T, R, \mathcal{L}\rangle$ from $\mathcal{C}$ (Note that it is legal for $\mathcal{F}$ to ask this signcryption from $\mathcal{C}) . \mathcal{F}$ constructs $\sigma^{*}=\operatorname{Signcryption}\left(m, \mathcal{L}^{\prime}=\right.$ $\left.\left\{I D_{1}, \ldots, I D_{k^{\prime}}\right\}, I D_{Y}, S_{1}, \ldots, S_{k^{\prime}}\right)$ as follows:

- As mentioned earlier let $\sigma=\langle c, T, R, \mathcal{L}\rangle$ be the signcryption on $m$ from $\mathcal{L}$ to $I D_{X}$. 
- $\mathcal{F}$ queries the private key $S_{Y}$, corresponding to $I D_{Y}$ (Since the receivers private key is available in the unforgeability game).

- $\mathcal{F}$ takes the value $R$ from $\sigma$ and computes $\omega^{*}=\hat{e}\left(R, S_{Y}\right)$ and computes a new value $c^{*}=m \oplus H_{2}\left(\omega^{*}\right)$.

- Computes $T^{*}=T-H_{3}(R)\left(\Sigma_{I D_{i} \in \mathcal{L}^{\prime}} S_{i}\right)$ and sets $R^{*}=R$

- Now, $\mathcal{F}$ produces $\sigma^{*}=\left\langle c^{*}, T^{*}, R^{*}, \mathcal{L}^{*}\right\rangle$ as a valid signcryption on the same message $m$ as $\sigma$ with $\mathcal{L}^{*}$ as the list of senders and $I D_{Y}$ as the receiver to the challenger.

We show that the new signcryption $\sigma^{*}=\left\langle c^{*}, T^{*}, R^{*}, \mathcal{L}^{*}\right\rangle$ is valid because it passes the verification with respect to the private key of the receiver $I D_{Y}$.

- Compute $\omega^{\prime}=\hat{e}\left(R^{*}, S_{Y}\right)=\hat{e}\left(R, S_{Y}\right)$.

- Retrieve the message $m^{\prime}=H_{2}\left(\omega^{\prime}\right) \oplus c^{*}$.

- The message $m^{\prime}$ obtained during unsigncryption is a valid message because $\hat{e}\left(T^{*}, P\right)=\hat{e}\left(R^{*}, H_{1}\left(m^{\prime}\right)\right)$ $\hat{e}\left(P_{p u b}, \sum_{j=1}^{n} Q_{j}\right)^{H_{3}\left(R^{*}\right)}$.

We show the correctness of the above check with respect to the forged signcryption.

$$
\begin{aligned}
\hat{e}\left(T^{*}, P\right) & =\hat{e}\left(\sum_{i=1}^{n} T_{i}, P\right) \\
& =\hat{e}\left(\sum_{i=1}^{n}\left\{x_{i} H_{1}\left(m^{\prime}\right)+H_{3}\left(R^{*}\right) S_{i}\right\}, P\right) \\
& =\hat{e}\left(\sum_{i=1}^{n}\left\{x_{i}\right\} H_{1}\left(m^{\prime}\right), P\right) \hat{e}\left(\sum_{i=1}^{n}\left\{H_{3}\left(R^{*}\right) s Q_{i}\right\}, P\right) \\
& =\hat{e}\left(H_{1}\left(m^{\prime}\right), \sum_{i=1}^{n}\left\{x_{i} P\right\}\right) \hat{e}\left(H_{3}\left(R^{*}\right) \sum_{i=1}^{n}\left\{Q_{i}\right\}, s P\right) \\
& =\hat{e}\left(H_{1}\left(m^{\prime}\right), R\right) \hat{e}\left(\sum_{i=1}^{n}\left\{Q_{i}\right\}, P_{p u b}\right)^{H_{3}\left(R^{*}\right)}
\end{aligned}
$$

Remark 2: Informally, this forgery is possible because there is no binding between the signature component $T_{i}$ and the receiver identity $I D_{X}$ and the other senders in list $\mathcal{L}$ involved in the generation of signcryption $\sigma$. The component $T_{i}$ acts as a signature because it is computed with the sender's private keys. So, the forger $\mathcal{F}$ can alter the receiver and generate the new signcryption $\sigma^{\prime}$ by changing the component $c$, the senders list and the receiver. The component $c^{\prime}$ can be computed using the private key of the new receiver, which is known to $\mathcal{F}$. (Note: It is well known that during unforgeability game for signcryption, $\mathcal{F}$ has access to the receiver's private key in order to ensure insider security.)

\section{Improved Identity Based Multi-Signcryption Scheme (I-IBMSC)}

The scheme by Jianhong et al. [16] can be fixed by modifying it suitably so that it satisfies the necessary conditions outlined by An, Dodis and Rabin [1]. In this section, we present a possible fix for [16]. We also prove the indistinguishability against adaptive chosen ciphertext attack (IND-CCA2) for confidentiality and existential unforgeablity against chosen message attack (EUF-CMA) for unforgeability of I-IBMSC in the random oracle model. These are the strongest security notions for any identity based multi-signcryption scheme. Our modifications are simple and subtle and the formal proofs are non-trivial.

\subsection{The Scheme (I-IBMSC)}

The improved scheme has the following four algorithms as in JJ-IBMSC [16].

1. Setup: This algorithm is similar to that of the Setup algorithm in JJ-IBMSC with a new definition for the hash function $H_{2}$. We define $H_{2}:\{0,1\}^{*} \rightarrow\{0,1\}^{l}$ in I-IBMSC. We also add one more hash function $H_{4}$, which is defined as $H_{4}:\{0,1\}^{*} \rightarrow \mathbb{G}_{1}$.

2. Key Extract: This algorithm is identical to that of the Key Extract algorithm in JJ-IBMSC.

3. Signcryption: Given a message $m$, a receiver identity $I D_{X}$ and a list of $n$ sender identities $\mathcal{L}=$ $\left\{I D_{1}, \ldots, I D_{n}\right\}$, each sender indexed $i$, for $i=1$ to $n$ executes the following steps:

- Chooses $x_{i} \in \mathbb{Z} q^{*}$ at random and computes $R_{i}=x_{i} P$.

- Computes $\omega_{i}=\hat{e}\left(P_{p u b}, Q_{X}\right)^{x_{i}}$.

- Sends $\left\langle R_{i}, \omega_{i}\right\rangle$ to all other senders in the list $\mathcal{L}$, through a secure channel.

After receiving all other senders $\left\langle R_{i}, \omega_{i}\right\rangle$ values, each sender continues to do the following;

- Computes $\omega=\prod_{j=1}^{n} \omega_{j}$ and $h_{2}=H_{2}\left(\omega, \mathcal{L}, I D_{X}\right)$.

- Sets $c=h_{2} \oplus m$, computes $R=\sum_{j=1}^{n} R_{j}, M=H_{4}\left(m, \omega, R, \mathcal{L}, I D_{X}\right)$ and $h_{3}=H_{3}\left(R, \mathcal{L}, I D_{X}\right)$. 
- Computes $T_{i}=x_{i} M+h_{3} S_{i}$.

Now, each sender $I D_{i}$ sends their corresponding $T_{i}, c$ and $R$ values to a clerk (Note that one of the senders in the list $\mathcal{L}$ may act as clerk), who checks whether $c$ and $R$ values sent by all senders are identical; if so, the clerk computes $T=\sum_{j=1}^{n} T_{j}$ and outputs the resultant signcryption as $\sigma=\langle c, T, R, \mathcal{L}\rangle$.

4. Unsigncryption: Given $\sigma=\langle c, T, R, \mathcal{L}\rangle$, the receiver with identity $I D_{X}$ does the following to unsigncrypt it:

- Computes $\omega^{\prime}=\hat{e}\left(R, S_{X}\right)$.

- Computes $h_{2}^{\prime}=H_{2}\left(\omega^{\prime}, \mathcal{L}, I D_{X}\right)$ and retrieves the message $m^{\prime}=h_{2}^{\prime} \oplus c$.

- Computes $M^{\prime}=H_{4}\left(m^{\prime}, \omega^{\prime}, R, \mathcal{L}, I D_{X}\right)$ and $h_{3}^{\prime}=H_{3}\left(R, \mathcal{L}, I D_{X}\right)$.

- Accepts the message $m^{\prime}$ if $\hat{e}(T, P) \stackrel{?}{=} \hat{e}\left(R, M^{\prime}\right) \hat{e}\left(P_{p u b}, \sum_{j=1}^{n}\left\{Q_{j}\right\}\right)^{h_{3}^{\prime}}$.

Correctness: In order to prove the correctness of I-IBMSC we prove the consistency of the check $\hat{e}(T, P) \stackrel{?}{=}$ $\hat{e}\left(R, M^{\prime}\right) \hat{e}\left(P_{p u b}, \sum_{j=1}^{n} Q_{j}\right)^{h_{3}^{\prime}}$ below:

$$
\begin{aligned}
\hat{e}(T, P) & =\hat{e}\left(\sum_{j=1}^{n} T_{j}, P\right) \\
& =\hat{e}\left(\sum_{j=1}^{n}\left\{x_{j} M^{\prime}+h_{3}^{\prime} S_{j}\right\}, P\right) \\
& =\hat{e}\left(\sum_{j=1}^{n}\left\{x_{j}\right\} M^{\prime}, P\right) \hat{e}\left(\sum_{j=1}^{n}\left\{h_{3}^{\prime} s Q_{j}\right\}, P\right) \\
& =\hat{e}\left(M^{\prime}, \sum_{j=1}^{n}\left\{x_{j} P\right\}\right) \hat{e}\left(h_{3}^{\prime} \sum_{j=1}^{n}\left\{Q_{j}\right\}, s P\right) \\
& =\hat{e}\left(M^{\prime}, R\right) \hat{e}\left(\sum_{j=1}^{n}\left\{Q_{j}\right\}, P_{p u b}\right)^{h_{3}^{\prime}}
\end{aligned}
$$

Note that the acceptance test is same as that of Jianhong et al.'s [16] but the change in the definition of the hash functions $H_{2}, H_{3}$ and the introduction of the new hash function $H_{4}$ provide the needed security for our system.

\subsection{Security of the Scheme (I-IBMSC)}

We prove the security of I-IBMSC with respect to confidentiality (IND-I-IBMSC-CCA2) and unforgeability (EUF-I-IBMSC-CMA) in this section.

\section{Confidentiality:}

Theorem 1. If an IND-I-IBMSC-CCA2 adversary $\mathcal{A}$ has an advantage $\epsilon$ against I-IBMSC scheme, asking $q_{H_{i}}(i=1,2,3,4)$ hash queries to random oracles $\mathcal{O}_{H_{i}}(i=1,2,3,4), q_{e}$ extract queries $\left(q_{e}=q_{e_{1}}+q_{e_{2}}\right.$, where $q_{e_{1}}$ and $q_{e_{2}}$ are the number of extract queries in the first phase and second phase respectively), $q_{s c}$ signcryption queries and $q_{u s}$ unsigncryption queries, then there exist an algorithm $\mathcal{C}$ that solves the $C B D H$ problem with advantage $\epsilon\left(\frac{1}{q_{H_{1}} q_{H_{2}}}\right)$.

Proof: Let $\mathcal{C}$ be a challenger, who is challenged with an instance of CBDHP say, $(P, a P, b P, c P) \in \mathbb{G}_{1}^{4}$ for unknown $a, b, c \in \mathbb{Z}_{q}^{*}$. The aim of $\mathcal{C}$ is to compute $\alpha=\hat{e}(P, P)^{a b c}$. Consider an adversary $\mathcal{A}$ who is capable of breaking the IND-I-IBMSC-CCA2 security of I-IBMSC. $\mathcal{C}$ can make use of $\mathcal{A}$ to solve the CBDHP instance with non-negligible advantage in polynomial time as described below.

It is assumed that in the IND-I-IBMSC-CCA2 game $\mathcal{A}$ queries $\mathcal{O}_{H_{1}}$ oracle with $I D$ before using $I D$ as input for any oracle query.

Setup: $\mathcal{C}$ starts the confidentiality game by setting up the system with Params $=\left\langle\kappa, \mathbb{G}_{1}, \mathbb{G}_{2}, P, \hat{e}, P_{\text {pub }}\right\rangle$, where $P_{p u b}=a P$ and models the hash functions $H_{i}$ 's as random oracles $\mathcal{O}_{H_{i}}$, for $i=1$ to 4 and sends the public parameters to $\mathcal{A}$.

Phase 1: $\mathcal{A}$ performs a series of queries to the oracles provided by $\mathcal{C}$. In-order to maintain the consistency of the responses given by the oracles $\mathcal{O}_{H_{i}}, \mathcal{C}$ maintain lists $L_{i}$, for $i=1$ to 4 . The oracles provided by $\mathcal{C}$ and the responses given to the corresponding queries by $\mathcal{C}$ are described below:

Oracle $\mathcal{O}_{H_{1}}\left(I D_{i}\right)$ : We will make a simplifying assumption that $\mathcal{A}$ queries the $\mathcal{O}_{H_{1}}$ oracle with distinct identities in each query. There is no loss of generality due to this assumption, because, if the same identity is repeated, by definition the oracle consults the list $L_{1}$ and gives the same response. Thus, we 
assume that $\mathcal{A}$ asks $q_{H_{1}}$ distinct queries for $q_{H_{1}}$ distinct identities. Among this $q_{H_{1}}$ identities, a random identity has to be selected as target identity and it is done as follows.

$\mathcal{C}$ selects a random index $\gamma$, where $1 \leq \gamma \leq q_{H_{1}}$. $\mathcal{C}$ does not reveal $\gamma$ to $\mathcal{A}$. When $\mathcal{A}$ generates the $\gamma^{\text {th }}$ query on $I D_{\gamma}, \mathcal{C}$ fixes $I D_{\gamma}$ as target identity for the challenge phase and $\mathcal{C}$ responds to $\mathcal{A}$ as follows:

- If $i=\gamma, \mathcal{C}$ sets $Q_{\gamma}=b P$, returns $Q_{\gamma}$ as the response to the query and stores $\left\langle I D_{\gamma}, Q_{\gamma}, *\right\rangle$ in the list $L_{1}$. Here, $\mathcal{C}$ does not know $b$ since $\mathcal{C}$ uses the $b P$ value given in the instance of the CBDHP.

- For all other queries, $\mathcal{C}$ chooses $y_{i} \in_{R} Z_{q}^{*}$ and sets $Q_{i}=y_{i} P$ and stores $\left\langle I D_{i}, Q_{i}, y_{i}\right\rangle$ in the list $L_{1}$.

$\mathcal{C}$ returns $Q_{i}$ to $\mathcal{A}$. (Note that as the identities are assumed to be distinct, for each query, we create distinct entry and add in the list $L_{1}$ ).

Oracle $\mathcal{O}_{H_{2}}\left(\omega, \mathcal{L}, I D_{X}\right)$ : When $\mathcal{A}$ makes this query, $\mathcal{C}$ checks for the tuple $\left\langle\omega, \mathcal{L}, I D_{X}, h_{2}\right\rangle$ in the list $L_{2}$. If an entry was found then returns the corresponding $h_{2}$ as the response else chooses $h_{2} \in_{R}\{0,1\}^{l}$ and adds the tuple $\left\langle\omega, \mathcal{L}, I D_{X}, h_{2}\right\rangle$ to the list $L_{2}$ and returns $h_{2}$ as the response to $\mathcal{A}$.

Oracle $\mathcal{O}_{H_{3}}\left(R, \mathcal{L}, I D_{X}\right)$ : When $\mathcal{A}$ makes this query, $\mathcal{C}$ checks for the tuple $\left\langle R, \mathcal{L}, I D_{X}, h_{3}\right\rangle$ in the list $L_{3}$. If an entry was found then returns the corresponding $h_{3}$ as the response else chooses $h_{3} \in_{R} \mathbb{Z}_{q}^{*}$ and adds the tuple $\left\langle R, \mathcal{L}, I D_{X}, h_{3}\right\rangle$ to the list $L_{3}$ and returns $h_{3}$ as the response to $\mathcal{A}$.

Oracle $\mathcal{O}_{H_{4}}\left(m, \omega, R, \mathcal{L}, I D_{X}\right)$ : When $\mathcal{A}$ makes this query, $\mathcal{C}$ checks for the tuple $\left\langle m, \omega, R, \mathcal{L}, I D_{X}, z, M\right\rangle$ in the list $L_{4}$. If an entry was found then returns the corresponding $M$ as the response else chooses $z \in \mathbb{Z}_{q}^{*}$ randomly and computes $M=z P_{p u b} . \mathcal{C}$ now adds the tuple $\left\langle m, \omega, R, \mathcal{L}, I D_{X}, z, M\right\rangle$ to the list $L_{4}$ and returns $M$ as the response to $\mathcal{A}$.

Oracle $\mathcal{O}_{\text {KeyExtract }}\left(I D_{i}\right): \mathcal{A}$ chooses an identity $I D_{i}$ and queries the corresponding private key $S_{i}$ to this oracle (Note that $\mathcal{A}$ should have performed the $\mathcal{O}_{H_{1}}\left(I D_{i}\right)$ query before performing this query). $\mathcal{C}$ responds to this query as follows:

- If $I D_{i}=I D_{\gamma}$, then $\mathcal{C}$ aborts.

- Else, $\mathcal{C}$ retrieves $y_{i}$ corresponding to $I D_{i}$ from $L_{1}$, computes $S_{i}=y_{i}(a P)=a\left(y_{i} P\right)=a Q_{i}$ and returns $S_{i}$ to $\mathcal{A}$.

Oracle $\mathcal{O}_{\text {Signcryption }}\left(m, \mathcal{L}, I D_{X}\right):$ Here, $m$ is the message to be signcrypted, $\mathcal{L}$ is the list of identities of $n$ senders and $I D_{X}$ is the identity of the receiver. If $I D_{\gamma} \notin \mathcal{L}$, then $\mathcal{C}$ can generate the signcryption on the message $m$ because $\mathcal{C}$ knows the private key of all senders belonging to $\mathcal{L}$.

- If $I D_{\gamma} \in \mathcal{L}$ then $\mathcal{C}$ performs the following:

- Chooses $z, r, h_{3} \in_{R} \mathbb{Z}_{q}^{*}$.

- Computes $M=z\left(P_{p u b}\right), R=\left(\frac{1}{z}\right)\left(r P-h_{3} \sum_{I D_{i} \in \mathcal{L}} Q_{i}\right)$.

- Stores the tuple $\left\langle R, \mathcal{L}, I D_{X}, h_{3}\right\rangle$ to the list $L_{3}$.

- Computes $T=r P_{p u b}$.

- Stores $\left\langle m, \omega, R, \mathcal{L}, I D_{X}, z, M\right\rangle$ in list $L_{4}$. (Note: If this tuple is already available in list $L_{4}$ then $\mathcal{C}$ repeats the above steps with different values of $r$ and $z$.)

- Compute $\omega=\hat{e}\left(R, S_{X}\right)$ and $c=m \oplus \mathcal{O}_{H_{2}}\left(\omega, \mathcal{L}, I D_{X}\right)$.

Now, $\mathcal{C}$ returns the signcryption on message $m$ as $\sigma=\langle c, T, R, \mathcal{L}\rangle$ to $\mathcal{A}$.

We show that the signcryption $\sigma=\langle c, T, R, \mathcal{L}\rangle$ produced by $\mathcal{C}$ passes the validity test. The validity check done during unsigncryption is $\hat{e}(T, P) \stackrel{?}{=} \hat{e}(R, M) \hat{e}\left(P_{p u b}, \sum_{i=1}^{n} Q_{i}\right)^{h_{3}}$

$$
\begin{aligned}
\text { R.H.S } & =\hat{e}(R, M) \hat{e}\left(P_{p u b}, \sum_{i=1}^{n} Q_{i}\right)^{h_{3}} \\
& =\hat{e}\left(z P_{p u b}, \frac{1}{z}\left(r P-h_{3} \sum_{I D_{i} \in \mathcal{L}} Q_{i}\right)\right) \hat{e}\left(P_{p u b}, \sum_{i=1}^{n} Q_{i}\right)^{h_{3}} \\
& =\hat{e}\left(P_{P u b}, r P\right) \hat{e}\left(P_{p u b}, \sum_{i=1}^{n} Q_{i}\right)^{h_{3}} \\
& =\hat{e}\left(P_{P u b}, r P\right) \hat{e}\left(P_{p u b},-\sum_{i=1}^{n} Q_{i}\right)^{h_{3}} \hat{e}\left(P_{p u b}, \sum_{i=1}^{n} Q_{i}\right)^{h_{3}} \\
& =\hat{e}\left(P_{P u b}, r P\right) \\
& =\hat{e}\left(r P_{p u b}, P\right) \\
& =\hat{e}(T, P) \\
& =\text { L.H.S }
\end{aligned}
$$


It is clear that L.H.S=R.H.S and thus $\sigma$ is a valid signcryption on message $m$ with $\mathcal{L}$ as the sender list, $I D_{\gamma} \in \mathcal{L}$ and $I D_{X}$ as the receiver.

Oracle $\mathcal{O}_{\text {Unsigncryption }}\left(\sigma, \mathcal{L}, I D_{X}\right)$ : Here, $\sigma$ is the signcryption, $\mathcal{L}$ is the list of identities of $n$ senders and $I D_{X}$ is the receiver identity. To respond to this query, $\mathcal{C}$ checks whether $I D_{X} \stackrel{?}{=} I D_{\gamma}$.

- If $I D_{X} \neq I D_{\gamma}, \mathcal{C}$ proceeds as per the normal unsigncryption algorithm, since $\mathcal{C}$ knows the private key of the receiver $I D_{X}$.

- If $I D_{X}=I D_{\gamma}$ then $\mathcal{C}$ computes $h_{3}=\mathcal{O}_{H_{3}}\left(R, \mathcal{L}, I D_{X}\right)$ and performs the following to unsigncrypt $\sigma$ :

1. Let $\Delta$ be the set of pairs $\left(\omega, h_{2}\right)$ from the list $L_{2}$ corresponding to $\left(\mathcal{L}, I D_{X}\right) \in \sigma$. (The tuples in $L_{2}$ will be of the form $\left\langle\omega, \mathcal{L}, I D_{X}, h_{2}\right\rangle$.)

2. For each $\left(\omega, h_{2}\right) \in \Delta, \mathcal{C}$ performs the following,

(a) Computes $m^{\prime}=c \oplus h_{2}$.

(b) Computes $M=\mathcal{O}_{H_{4}}\left(m^{\prime}, \omega, \mathcal{L}, I D_{X}\right)$.

(c) Checks whether $\hat{e}(M, R)^{1 / z} \stackrel{?}{=} \omega$ and $\hat{e}(T, P) \stackrel{?}{=} \hat{e}(M, R) \hat{e}\left(P_{p u b}, \sum_{I D_{i} \in \mathcal{L}} Q_{i}\right)^{h_{3}}$. (Note that $z$ is retrieved from the tuples in the list $L_{4}$ corresponding to $\left(m^{\prime}, \omega, \mathcal{L}, I D_{X}\right)$, which is of the form $\left\langle m^{\prime}, \omega, \mathcal{L}, I D_{X}, z, M\right\rangle \in L_{4}$.)

(d) If true, return $m^{\prime}$.

3. If the test in step $2(\mathrm{c})$ fails for all $\left(\omega, h_{2}\right) \in \Delta, \mathcal{C}$ returns "invalid".

Challenge: At the end of Phase 1, $\mathcal{A}$ generates and sends to $\mathcal{C}$, two plaintexts $m_{0}$ and $m_{1}$ of equal length, a sender list $\mathcal{L}=\left\{I D_{1}, \ldots, I D_{\bar{n}}\right\}$ and the receiver identity $I D_{X}$. Note that $\mathcal{A}$ should not have queried the private key corresponding to $I D_{X}$ in Phase 1. $\mathcal{C}$ checks whether $I D_{X}=I D_{\gamma}$. If not, $\mathcal{C}$ aborts, else, $\mathcal{C}$ chooses a bit $b \in_{R}\{0,1\}$ and computes the challenge signcryption $\sigma^{*}$ on the message $m_{b}$ with $\mathcal{L}$ as the group of senders and $I D_{X}$ as the receiver by performing the following:

$-\mathcal{C}$ sets $R=c P$, chooses randomly $T \in \mathbb{G}_{1}$ and $c \in\{0,1\}^{l}$

Now, $\mathcal{C}$ outputs the signcryption on the message $m_{b}$ as $\sigma=\langle c, T, R, \mathcal{L}\rangle$.

Phase 2: $\mathcal{A}$ can perform polynomially bounded number of queries adaptively again as in Phase 1 with the restriction that $\mathcal{A}$ cannot query

- The key extract oracle for the private key of $I D_{X}$.

- The unsigncryption oracle with the challenge signcryption $\sigma^{*}, I D_{X}$.

Guess: At the end of Phase 2, $\mathcal{A}$ outputs a guess $b^{\prime}$ to $\mathcal{C}$. Similar to the argument in [5], $\mathcal{C}$ ignores the response by $\mathcal{A}$, picks a random $\omega$ from the list $L_{2}$ and returns it as the solution to the CBDHP instance. Since the challenge ciphertext $\sigma^{*}$ given to $\mathcal{A}$ is randomly distributed in the ciphertext space, $\mathcal{A}$ cannot gain any advantage in the IND-I-IBMSC-CCA2 game. Thus, any adversary that has advantage $\epsilon$ in the real IND-I-IBMSC-CCA2 game must necessarily recognize with probability at least $\epsilon$ that the challenge ciphertext provided by $\mathcal{C}$ is incorrect. For $\mathcal{A}$ to find that $\sigma^{*}$ is not a valid ciphertext, $\mathcal{A}$ should have queried the $\mathcal{O}_{H_{2}}$ oracle with $\omega=\hat{e}\left(R, S_{\gamma}\right)$ (Since the receiver in $\sigma^{*}$ is $\left.I D_{X}=I D_{\gamma}\right)$. Here $S_{\gamma}$ is the private key of the target identity and it is $a\left(Q_{\gamma}\right)=a b P$. Also, $\mathcal{C}$ has set $R=c P$. Hence $\omega=\hat{e}\left(R, S_{\gamma}\right)=\hat{e}(c P, a b P)=\hat{e}(P, P)^{a b c}$. Therefore, one of the entries in the list $L_{2}$ should be the value $\hat{e}(P, P)^{a b c}$. With probability $\frac{1}{q_{H_{2}}}$, the value of $\omega$ chosen by $\mathcal{C}$ from list $L_{2}$ will be the solution to CBDHP instance.

Now, we analyze the probability of success of $\mathcal{C}$. The events in which $\mathcal{C}$ aborts the IND-I-IBMSC-CCA2 game are,

1. $E_{1}$ - when $\mathcal{A}$ queries the private key of the target identity $I D_{\gamma}$ and $\operatorname{Pr}\left[E_{1}\right]=\frac{q_{e_{1}}}{q_{H_{1}}}$.

2. $E_{2}$ - when $\mathcal{A}$ does not choose the target identity $I D_{\gamma}$ as the receiver during the challenge and $\operatorname{Pr}\left[E_{2}\right]=\left(1-\frac{1}{q_{H_{1}}-q_{e_{1}}}\right)$.

The probability that, $\mathcal{C}$ does not abort the IND-I-IBMSC-CCA2 game is given by

$$
\left(\operatorname{Pr}\left[\neg E_{1} \wedge \neg E_{2}\right]\right)=\left(1-\frac{q_{e_{1}}}{q_{H_{1}}}\right)\left(\frac{1}{q_{H_{1}}-q_{e_{1}}}\right)=\frac{1}{q_{H_{1}}}
$$

The probability that, the $\omega$ chosen randomly from $L_{2}$ by $\mathcal{C}$, being the solution to CBDHP is $\left(\frac{1}{q_{H_{2}}}\right)$. Therefore, the probability of $\mathcal{C}$ solving CBDHP is given by, 


$$
\operatorname{Pr}\left[\mathcal{C}\left(P, a P, b P, c P \mid a, b, c \in_{R} \mathbb{Z}_{q}^{*}\right)=\hat{e}(P, P)^{a b c}\right]=\epsilon\left(\frac{1}{q_{H_{1}} q_{H_{2}}}\right)
$$

Since $\epsilon$ is non-negligible, the probability of $\mathcal{C}$ solving CBDHP is also non-negligible.

\section{Unforgeability:}

Theorem 2. If an EUF-I-IBMSC-CMA forger $\mathcal{F}$ exists against I-IBMSC scheme, asking $q_{H_{i}}(i=1,2,3$, 4) hash queries to random oracles $H_{i}\left(i=1\right.$, 2, 3, 4), $q_{e}$ extract secret key queries, $q_{\text {sc }}$ signcryption queries and $q_{u s}$ unsigncryption queries, then there exist an algorithm $\mathcal{C}$ that solves the CDHP with advantage.

Proof: Let $\mathcal{C}$ be a challenger who is challenged with an instance of CDH problem say, $(P, a P, b P) \in \mathbb{G}_{1}^{3}$ for unknown $a, b \in \mathbb{Z}_{q}^{*}$. The aim of $\mathcal{C}$ is to compute the value $a b P$. Consider a forger $\mathcal{F}$ who is capable of breaking the EUF-I-IBMSC-CMA security of I-IBMSC. $\mathcal{C}$ can make use of $\mathcal{F}$ to solve the CDH problem instance with non-negligible advantage in polynomial time as described below.

Setup: $\mathcal{C}$ starts the unforgeability game by setting up the system with $P_{p u b}=a P$, models the hash functions $H_{i}$ 's as random oracles $\mathcal{O}_{H_{i}}$, for $i=1$ to 4 and sends the public parameters Params $=\left\langle\kappa, \mathbb{G}_{1}, \mathbb{G}_{2}, P, \hat{e}, P_{\text {pub }}\right\rangle$ to $\mathcal{F}$. The elements of Params are set identical to that of the $\mathrm{CDH}$ instance, $\mathcal{C}$ has received.

It is assumed in the EUF-I-IBMSC-CMA game that $\mathcal{A}$ queries $\mathcal{O}_{H_{1}}$ oracle with $I D$ before using $I D$ as input for any other query.

Training Phase: $\mathcal{F}$ performs a series of queries to the oracles provided by $\mathcal{C}$. In-order to maintain the consistency of the responses given by the oracles $\mathcal{O}_{H_{i}}, \mathcal{C}$ maintain lists $L_{i}$, for $i=1$ to 4 . The oracles $\mathcal{O}_{H_{i}}, i=1,2,3$ and $\mathcal{O}_{\text {KeyExtract }}$ provided by $\mathcal{C}$ and the responses given to the corresponding queries by $\mathcal{C}$ are identical to that of the IND-I-IBMSC-CCA2 game. The description of the other oracles are given below,

Oracle $\mathcal{O}_{H_{4}}\left(m, \omega, R, \mathcal{L}, I D_{X}\right):$ When $\mathcal{A}$ makes this query, $\mathcal{C}$ checks for the tuple $\left\langle m, \omega, R, \mathcal{L}, I D_{X}, z, M\right\rangle$ in the list $L_{4}$. If an entry was found then returns the corresponding $M$ as the response else chooses $z \in \mathbb{Z}_{q}^{*}$ randomly and if $I D_{X}=I D_{\gamma}, \mathcal{C}$ computes $M=z P$ else computes $M=z P_{p u b}$. $\mathcal{C}$ now adds the tuple $\left\langle m, \omega, R, \mathcal{L}, I D_{X}, z, M\right\rangle$ to the list $L_{4}$ and returns $M$ as the response to $\mathcal{A}$.

Oracle $\mathcal{O}_{\text {Signcryption }}\left(m, \mathcal{L}, I D_{X}\right):$ Here, $m$ is the message to be signcrypted, $\mathcal{L}$ is the list of identities of $n$ senders and $I D_{X}$ is the receiver identity (Note that the receiver identity can also be the target identity $\left.I D_{X}\right)$. Even in the case of $I D_{X}=I D_{\gamma}, \mathcal{C}$ can generate the signcryption on the message $m$ because $\mathcal{C}$ knows the private key of all senders belonging to $\mathcal{L}$. In order to respond to this query, $\mathcal{C}$ checks for each $I D_{i} \in \mathcal{L}$, whether $I D_{i}=I D_{\gamma}$. If $I D_{\gamma} \notin \mathcal{L}, \mathcal{F}$ follows the signcrypt protocol as $\mathcal{C}$ knows the private key of all senders in $\mathcal{L}$. If $I D_{\gamma} \in \mathcal{L}$ then $\mathcal{C}$ performs the following:

- Chooses $z, h_{3}$ randomly from $\mathbb{Z}_{q}^{*}$

- Computes $M=z Q_{\gamma}$ and $R=\left(\frac{-h_{3}}{z}\right) P_{p u b}$.

- Computes $\omega=\hat{e}\left(R, S_{X}\right)$ and $c=m \oplus \mathcal{O}_{H_{2}}\left(\omega, \mathcal{L}, I D_{X}\right)$.

- Computes $T=h_{3}\left(\sum_{I D_{i} \in \mathcal{L} \wedge I D_{i} \neq I D_{\gamma}} S_{i}\right)$.

- Stores $\left\langle m, \omega, R, \mathcal{L}, I D_{X}, z, M\right\rangle$ in list $L_{4}$. (Note: If this tuple is already available in list $L_{4}$ then $\mathcal{C}$ repeats the above steps with different values.)

Now, $\mathcal{C}$ stores $\left\langle m, \sigma=\langle c, T, R, \mathcal{L}\rangle, I D_{X}\right\rangle$ in list $L_{5}$ returns the signcryption on message $m$ as $\sigma$ to $\mathcal{F}$.

We now show that the signcryption $\sigma=\langle c, T, R, \mathcal{L}\rangle$ produced by $\mathcal{C}$ is valid because it passes the verification test $\hat{e}(T, P) \stackrel{?}{=} \hat{e}(R, M) \hat{e}\left(P_{p u b}, \sum_{i=1}^{n} Q_{i}\right)^{h_{3}}$ as shown below.

$$
\begin{aligned}
\text { R.H.S } & =\hat{e}(R, M) \hat{e}\left(P_{p u b}, \sum_{I D_{i} \in L} Q_{i}\right)^{h_{3}} \\
& =\hat{e}\left(\left(\frac{-h_{3}}{z}\right) P_{p u b}, z P\right) \hat{e}\left(P_{p u b}, \sum_{I D_{i} \in L} Q_{i}\right)^{h_{3}} \\
& =\hat{e}\left(P_{p u b}, \sum_{I D_{i} \in L \wedge I D_{i} \neq I D_{\gamma}} Q_{i}\right)^{h_{3}} \\
& =\hat{e}\left(P, h_{3} \sum_{I D_{i} \in L \wedge I D_{i} \neq I D_{\gamma}} S_{i}\right) \\
& =\hat{e}(T, P) \\
& =\text { L.H.S }
\end{aligned}
$$


It is clear that L.H.S=R.H.S and thus $\sigma$ is a valid signcryption on message $m$ with $\mathcal{L}$ as the sender list, $I D_{\gamma} \in \mathcal{L}$ and $I D_{X}$ as the receiver.

Oracle $\mathcal{O}_{\text {Unsigncryption }}\left(\sigma, \mathcal{L}, I D_{X}\right)$ : Here, $\sigma$ is the signcryption, $\mathcal{L}$ is the list of identities of $n$ senders and $I D_{X}$ is the receiver identity. To respond to this query, $\mathcal{C}$ does the following.

- If $I D_{X} \neq I D_{\gamma}$ then $\mathcal{C}$ knows the private key of $I D_{X}$ and can perform the unsigncryption as per the protocol.

- If $I D_{X}=I D_{\gamma}$, then $\mathcal{C}$ performs the following to unsigncrypt $\sigma$

- If $\sigma \in L_{5}$, then $\mathcal{C}$ returns $m$ to $\mathcal{F}$,

- Otherwise, $\mathcal{C}$ does the following:

1. Computes $h_{3}=\mathcal{O}_{H_{3}}\left(R, L, I D_{X}\right)$.

2. Let $\Delta$ be the set of pairs $\left(\omega, h_{2}\right)$ from the list $L_{2}$ corresponding to $\left(\mathcal{L}, I D_{X}\right) \in \sigma$. (The tuples in $L_{2}$ will be of the form $\left\langle\omega, \mathcal{L}, I D_{X}, h_{2}\right\rangle$.)

3. For each $\left(\omega, h_{2}\right) \in \Delta, \mathcal{C}$ performs the following,

(a) Computes $m^{\prime}=c \oplus h_{2}$.

(b) Computes $M=\mathcal{O}_{H_{4}}\left(m^{\prime}, \omega, \mathcal{L}, I D_{X}\right)$.

(c) Checks whether $\hat{e}(M, R)^{1 / z} \stackrel{?}{=} \omega$ and $\hat{e}(T, P) \stackrel{?}{=} \hat{e}(M, R) \hat{e}\left(P_{p u b}, \sum_{I D_{i} \in \mathcal{L}} Q_{i}\right)^{h_{3}}$. (Note that $z$ is retrieved from the tuples in the list $L_{4}$ corresponding to $\left(m^{\prime}, \omega, \mathcal{L}, I D_{X}\right)$, which is of the form $\left.\left\langle m^{\prime}, \omega, \mathcal{L}, I D_{X}, z, M\right\rangle \in L_{4}\right)$.

(d) If true, return $m^{\prime}$.

4. If the test in step 3(c) fails for all $\left(\omega, h_{2}\right) \in \Delta, \mathcal{C}$ returns "invalid".

Forgery: Eventually, $\mathcal{F}$ outputs a forged signcryption $\sigma^{*}=\left\langle c^{*}, T^{*}, R^{*}, \mathcal{L}\right\rangle$ on some message $m^{*}$ with $\mathcal{L}$ as the list of senders and an arbitrary receiver, say $I D_{X}$. The restriction in generating $\sigma^{*}$ is, $\mathcal{F}$ should not have generated $\sigma^{*}$ by querying the signcryption oracle $\mathcal{O}_{\text {Signcryption }}$ in any previous queries on the message $m^{*}$ with $\mathcal{L}$ as the list of senders and $I D_{X}$ as the receiver. $\mathcal{C}$ can very well unsigncrypt and verify the validity of the forged signcryption $\sigma^{*}$ because $\mathcal{C}$ knows the secret key of the receiver during the unforgeability game. If the forged signcryption $\sigma^{*}$ passes the verification and at least one of the identities in $\mathcal{L}$ say $I D_{A}$ was not the output of any previous key extract queries, then $\mathcal{C}$ can obtain the solution for the $\mathrm{CDH}$ problem instance by performing the following steps:

$-\mathcal{C}$ obtains $\omega^{*}$ and the message $m^{*}$ during unsigncryption.

- It then checks the list $L_{4}$ for the tuple of the form $\left\langle m^{*}, \omega^{*}, R^{*}, \mathcal{L}, I D_{X}, z, M\right\rangle$.

- Computes $\sum_{i=1}^{n}\left\{x_{i} M\right\}=\sum_{i=1}^{n} x_{i}(z P)=z \sum_{i=1}^{n}\left(x_{i}\right) P=z R$.

- Computes $X=T-\sum_{i=1}^{n}\left\{x_{i} M\right\}=\sum_{i=1}^{n} h_{3} S_{i}$.

- Computes $Y=\sum_{i=1}^{n}\left\{h_{3} S_{i}\right\}-\sum_{i=1, i \neq A}^{n} h_{3} S_{i}=h_{3} S_{A}$. This is possible because $\mathcal{C}$ can retrieve the value $h_{3}$ from the list $L_{3}$ which corresponds to the tuple $\left\langle R, \mathcal{L}, I D_{X}, h_{3}\right\rangle$ and $\mathcal{C}$ knows the private key corresponding to all the identities of the sender list $\mathcal{L}$ except $I D_{A}$.

Therefore, $\mathcal{C}$ can compute: $\left(h_{3}\right)^{-1} Y=\left(h_{3}\right)^{-1} h_{3} S_{A}$

$$
\begin{aligned}
& =\left(h_{3}\right)^{-1} h_{3} a b P \\
& =a b P
\end{aligned}
$$

Thus, $\mathcal{C}$ is capable of finding $a b P$ value which is the solution for the $\mathrm{CDH}$ problem instance. So, if there exists a forger who can forge a valid signcryption with non-negligible advantage, then there exists an algorithm to solve the $\mathrm{CDH}$ problem with non-negligible advantage. Since this is not possible, no forger can forge a valid signcryption with non-negligible advantage. Hence, I-IBMSC is secure against any EUF-I-IBMSC-CMA attack. Now we analyse the probability of success of $\mathcal{C}$ in solving the CDHP.

$$
\operatorname{Pr}\left[\mathcal{C}(a P, b P)=a b P \mid a, b \in \mathbb{Z}_{q}^{*}\right]=\operatorname{Pr}\left[\mathcal{F}\left(\sigma^{*}, I D_{X}\right)=\operatorname{Valid}\right] . \operatorname{Pr}[\neg \text { Abort }]
$$

The events in which $\mathcal{C}$ aborts are:

- $E_{1}-\mathcal{F}$ queries the private key of the target identity to the key extract oracle and $\operatorname{Pr}\left[E_{1}\right]=\left(\frac{q_{e}}{q_{H_{1}}}\right)$.

- $E_{2}-\mathcal{F}$ doesnot choose $I D_{\gamma}$ as sender in $\mathcal{L}$ which is used for forgery and $\operatorname{Pr}\left[E_{2}\right]=\left(\frac{q_{H_{1}}-q_{e}-n}{q_{H_{1}}-q_{e}}\right)=$ $\left(1-\frac{n}{q_{H_{1}}-q_{e}}\right)$. 
Therefore, the probability that $\mathcal{C}$ does not abort the game is given by

$$
\begin{aligned}
\operatorname{Pr}[\neg \text { Abort }] & =\operatorname{Pr}\left[\neg E_{1} \wedge \neg E_{2}\right] \\
& =\left(1-\frac{q_{e}}{q_{H_{1}}}\right)\left(1-\left(1-\frac{n}{q_{H_{1}}-q_{e}}\right)\right) \\
& =\left(\frac{q_{H_{1}}-q_{e}}{q_{H_{1}}}\right)\left(\frac{n}{q_{H_{1}}-q_{e}}\right) \\
& =\frac{n}{q_{H_{1}}}
\end{aligned}
$$

Thus, the probability of success of $\mathcal{C}$ is given by $\operatorname{Pr}\left[\mathcal{C}(a P, b P)=a b P \mid a, b \in \mathbb{Z}_{q}^{*}\right]=\epsilon \frac{n}{q_{H_{1}}}$

\section{Conclusion}

As the only existing identity based multi-signcryption scheme is cryptanalized for its confidentiality and unforgeability, we do not compare the efficiency of our scheme with any other scheme but we present the complexity figure of I-IBMSC scheme below:

\begin{tabular}{|c||c|c|c|c||c|c|c|c|}
\hline \multicolumn{1}{|c||}{ Scheme } & \multicolumn{4}{c||}{ Signcrypt } & \multicolumn{3}{c|}{ Designcrypt } \\
\cline { 2 - 8 } & $P A$ & $S M$ & $G E$ & $M G$ & $P A$ & $S M$ & $G E$ & $M G$ \\
\hline
\end{tabular}

Table-1: Complexity figure for I-IBMSC

PA - Pairing, SM - Scalar Multiplication, GE - Exponentiation in $\mathbb{G}_{2}, G M-$ Mapping to $\mathbb{G}_{1}$.

We have provided an improved identity based multi-signcryption scheme, which is an extension of Jianhong et al.'s scheme with the proper binding, that provides adequate security to the scheme. We have also proved the security of the improved scheme formally under the random oracle model.

\section{References}

1. Jee Hea An, Yevgeniy Dodis, and Tal Rabin. On the security of joint signature and encryption. In Advances in Cryptology - EUROCRYPT 2002, volume 2332 of Lecture Notes in Computer Science, pages 83-107. Springer, 2002.

2. Joonsang Baek, Ron Steinfeld, and Yuliang Zheng. Formal proofs for the security of signcryption. In Public Key Cryptography - PKC 2002, volume 2274 of Lecture Notes in Computer Science, pages 80-98. Springer, 2002.

3. Paulo S. L. M. Barreto, Benoît Libert, Noel McCullagh, and Jean-Jacques Quisquater. Efficient and provablysecure identity-based signatures and signcryption from bilinear maps. In Advances in Cryptology - ASIACRYPT 2005, volume 3788 of Lecture Notes in Computer Science, pages 515-532. Springer, 2005.

4. Mihir Bellare and Gregory Neven. Identity-based multi-signatures from rsa. In Topics in Cryptology - CT-RSA 200\%, volume 4377 of Lecture Notes in Computer Science, pages 145-162. Springer, 2007.

5. Xavier Boyen. Multipurpose identity-based signcryption (a swiss army knife for identity-based cryptography). In Advances in Cryptology - CRYPTO 2003, volume 2729 of Lecture Notes in Computer Science, pages 383-399. Springer, 2003.

6. Sherman S. M. Chow, Siu-Ming Yiu, Lucas Chi Kwong Hui, and K. P. Chow. Efficient forward and provably secure id-based signcryption scheme with public verifiability and public ciphertext authenticity. In ICISC, volume 2971 of Lecture Notes in Computer Science, pages 352-369. Springer, 2003.

7. Craig Gentry and Zulfikar Ramzan. Identity-based aggregate signatures. In Public Key Cryptography - PKC 2006, volume 3958 of Lecture Notes in Computer Science, pages 257-273. Springer, 2006.

8. Benoît Libert and Jean-Jacques Quisquater. Efficient signcryption with key privacy from gap diffie-hellman groups. In Public Key Cryptography - PKC 2004, volume 2947 of Lecture Notes in Computer Science, pages 187-200. Springer, 2004.

9. Benot Libert and Jean-Jacques Quisquater. A new identity based signcryption scheme from pairings. In In IEEE Information Theory Workshop, pages 155-158, 2003. 
10. John Malone-Lee. Identity-based signcryption. Cryptology ePrint Archive, Report 2002/098, 2002.

11. Shirow Mitomi and Atsuko Miyaji. A multisignature scheme with message flexibility, order flexibility and order verifiability. In Information Security and Privacy, ACISP - 2000, volume 1841 of Lecture Notes in Computer Science, pages 298-312. Springer, 2000.

12. Xiaolin Pang, Barbara Catania, and Kian-Lee Tan. Securing your data in agent-based p2p systems. In Eighth International Conference on Database Systems for Advanced Applications - DASFAA 2003, pages 55-64. IEEE Computer Society, 2003.

13. Seung-Hyun Seo and Sang-Ho Lee. A secure and flexible multi-signcryption scheme. In Computational Science and Its Applications, ICCSA - 2004, volume 3046 of Lecture Notes in Computer Science, pages 689-697. Springer, 2004.

14. Adi Shamir. Identity-based cryptosystems and signature schemes. In Advances in Cryptology, CRYPTO - 1984, volume 196 of Lecture Notes in Computer Science, pages 47-53. Springer, 1984.

15. Chik How Tan. On the security of signcryption scheme with key privacy. IEICE Transactions, volume 88A(4):pages 1093-1095, 2005.

16. Jianhong Zhang and Jian Mao. A novel identity-based multi-signcryption scheme. Computer Communications, volume 32(1):pages 14-18, 2009.

17. Yuliang Zheng. Digital signcryption or how to achieve cost(signature \& encryption) $<<\operatorname{cost}($ signature) + cost(encryption). In Advances in Cryptology, CRYPTO - 199\%, volume 1294 of Lecture Notes in Computer Science, pages 165-179. Springer, 1997. 